\title{
Posttraumatic headache in concussed young adult athletes
}

\begin{abstract}
Background: Approximately 170 million young adults participate in organized sports of which almost four million are diagnosed with a concussion annually. Of these approximately 1.1 million visits the emergency department and are treated for a form of mild traumatic brain injury of which concussion is encompassed. Approximately $86 \%$ of young adult athletes who sustain a concussion report posttraumatic headache (PTH). Diagnostic tests are unable to accurately diagnose PTH leading to increased utilization and healthcare costs.
\end{abstract}

Aim: To determine a valid and reliable tool, which assesses postconcussive symptoms leading to decreased morbidity and mortality rates, while decreasing the burden of increased associated healthcare costs and utilization and how such tool could be successfully implemented?

Method: A literature review using the GRADE criterion to determine unmet needs, validity and reliability of postconcussive tools, and how implementation of theoretical frameworks could improve outcomes.

Conclusion: The PCSS scale was proven valid, reliable, and to have a high PPV in accurately diagnosing concussive symptoms and may be able to predict protracted recovery courses in an acute care setting, such as the E.D. These scales and checklists can be implemented among a multidisciplinary team by using Rogers Diffusion of Innovation theory.
Volume 2 Issue 3 - 2017

\author{
Deanna Jung \\ Department of Nursing, California State University, USA
}

Correspondence: Deanna Jung, Department of Nursing,

California State University, USA,

Email dejung@Exchange.fullerton.edu

Received: January 28, 2017 | Published: February 10, 2017
Abbreviations: ED, emergency department; PTH, posttraumatic headache; CT, computerized tomography; CNS, clinical nurse specialist; PCSS, postconcussive symptoms scale; NP, nurse practitioner; NHL, national hockey league; GOAT, galveston orientation and amnesia test; PCSS, post concussive symptom scale

\section{Introduction}

Approximately 170 million young adults aged 16-27 participate in an organized sport. ${ }^{1}$ There are almost four million young athletes who are diagnosed with a concussion annually. ${ }^{2}$ Of these approximately 1.1 million young adults visit the emergency department (E.D.) and are treated for a form of mild traumatic brain injury of which concussion is encompassed with an additional 300,000 admitted to the hospital. ${ }^{1}$ Concussion is defined as a type of mild traumatic brain injury and these terms are used most often interchangeably. ${ }^{3}$ Headache is the number one complaint of which young adult athletes sustaining a concussion are seen medically. ${ }^{3}$

Young adult athletes are at increased risk of sustaining a mild traumatic brain injury as they are four times at greater risk than all other combined age groups. ${ }^{4}$ Approximately $86 \%$ of young adult athletes who sustain a concussion report posttraumatic headache (PTH). ${ }^{5}$ However, it is during this time of young adulthood that health behaviors are formed with the greatest impact being on health education and preventative care are derived. ${ }^{4}$

Young adults typically display poor judgment; a higher rate of impulsivity, as their frontal lobe develops at different rates and is not fully developed and vulnerable to injury. ${ }^{6}$ These characteristics of a young adult athlete increase their risk factors both psychologically and physiologically for sustaining a traumatic brain injury, specifically a concussion. ${ }^{6}$
Currently there are arguably different definitions of what constitutes a concussion among health professionals and across specialties. ${ }^{7}$ Concussions are not limited by demographic factors such as sex, age, race, or ethnicity. Concussions can and do occur across the continuum of care from infancy to the elderly. ${ }^{7}$ However, the largest proportion of concussions occurs in young adult athletes in organized sports. ${ }^{1}$ According to the International Conference in Sport a concussion is defined as a complex pathophysiological process that alters brain functioning, including: focal neurological deficits, alteration in mental state, ante grade or retrograde amnesia, or any period of loss of consciousness by either direct or indirect external force.

Posttraumatic headaches and accompanying symptoms occurring in the United States cost over 48 billion dollars to assess, diagnose, and manage annually. ${ }^{8}$ This figure includes the time of initial presentation through rehabilitation services rendered in protracted recovery courses. ${ }^{8}$ The rehabilitative services required in most cases of protracted PTH have included: physical and occupational therapy, physiatrists, pain specialists, and neuropsychologists. Unnecessary diagnostic tests are commonly completed on concussed athletes leading to delay and potentially incorrect diagnosis. ${ }^{7,9}$ Undiagnosed, underreported, or delayed diagnoses of concussions are common occurrences in the United States increasing associated costs among healthcare systems. ${ }^{9}$

Concussions and symptoms following predominately PTH are unidentifiable or not visualized using common neuro imaging such as a computerized tomography (CT) scan ${ }^{3}$ consequently increasing time to diagnosis and cost to the healthcare system. Currently, there is neither serum nor diagnostic testing that has the ability to accurately diagnose concomitant symptoms such as a PTH in concussed young 
adult athletes. ${ }^{3}$ Concussions that are undiagnosed, underreported, or have delayed diagnoses can lead to additional traumatic brain injury and damage in concussed young adult athletes. Concussed individuals are at increased incidence of post-concussive syndrome, second impact syndrome and even death should repeated injury occur without adequate duration to heal completely. ${ }^{10}$

Posttraumatic headache and the sequelae of symptoms can occur from direct or indirect injury to the head and can last upwards to a year causing the inability to return to normal daily activities from protracted symptoms and increased length of absence from work and school. The most common of these protracted symptoms commonly found in concussed athletes and post concussive syndrome is posttraumatic headache?

Identification of this population in order for primary prevention strategies to be implemented is the initial step to improve outcomes and decrease the burden of disease. ${ }^{11}$ Interventions aimed at primary prevention include recognition, education, pre-season neuro cognitive testing, and increasing awareness at both the high school and college levels in both male and female collegiate sports. Secondary prevention efforts are defined after which a concussion has occurred. These efforts are aimed at accurate and timely diagnosis, rest, and treatment efforts based on individual symptomology. Tertiary prevention efforts are aimed at timely referral to neuropsychologist to assess concussed patients with protracted symptoms. Efforts are made across the continuum of care for rehabilitation and timely return to work and school.

Current evidence based guidelines for a primary headache and one sustained secondarily differ in their treatment and management therapies. A secondary headache, such as one sustained from a concussion suggest a detailed history of the injury or insult and risk factors, early education to both athletes and their parents, prevention strategies, preventing further injury, techniques to manage stress, written instructions, a graded return to work, school, and activity, management of physical, cognitive and behavioral symptoms, physical rehabilitation, alternative modalities, and the importance of follow up with providers. ${ }^{12}$ Individualization of treatment plans are based on specific symptoms and duration of those symptoms. ${ }^{12}$

Of these guidelines, a detailed history of the injury and risk factors, education, and a graded return to school, work, and activity are most important. Initial and protracted symptoms must be determined in order to adequately and efficiently diagnose concussions and the subtle symptoms that follow, most importantly a posttraumatic headache.

Worsening headaches in concussed athletes is considered a red flag. Although PTHs are present in over $80 \%$ of concussed patients, it is easily underreported. ${ }^{10}$ Implementation of screening and education in all aspects of the healthcare system should be sought to lessen the burden of the symptoms and disease in this population so they can be diminished and effectively managed.

Emergency room physicians and nurses need to be educated on symptoms of concussion, post concussive syndrome, and second impact syndrome. Trauma centers typically receive thousands of trauma patients annually. ${ }^{10}$ It is not uncommon for a trauma center to receive a young adult that has undergone a direct or indirect external force blunt trauma causing a concussion. When young adult athletes present to the E.D. of trauma centers identification and correct diagnosis is essential to obtaining the correct treatment modalities. Nurses are the first person a patient encounters in the E.D. This is the patients' gateway into the healthcare system. They are also the last person the patient encounters prior to being discharged. With increased use of acute care NPs in the E.D., nurses may be the only healthcare providers that the patient encounters during their short stay.

The American College of Surgeons ${ }^{13}$ mission is to implement meaningful programs at trauma centers that include education, professional development, standards of care, and assessment of outcomes in local communities. In essence, designated trauma centers are required to give back to their communities in these various areas. Therefore, the implementation of a protocol to screen for the most common symptoms, PTH, of a traumatic brain injury, mainly concussions, could be developed to decrease morbidity and mortality rates associated with concussion and those extending to protracted recovery courses or worse second impact syndrome. This protocol could easily be implemented by the clinical nurse specialist (CNS) with the aid of the development of a postconcussive symptoms scale (PCSS) by the nurse practitioner (NP) to be used in the E.D. for young adult concussed athletes.

\section{Theoretical frameworks}

Headache is the leading symptom following a traumatic injury. Posttraumatic headaches are found in approximately $79-100 \%$ of athletes in organized sports immediately following a concussion. In an effort to decrease morbidity associated with postconcussive headaches in young adult athletes two nursing models can be used succinctly. Bandura's Self Efficacy Model may be beneficial when educating young adult athletes and their parents. Also, Rogers Diffusion Model can be utilized when designing and implementing a protocol, or PCSS, into practice in the E.D. by advance practice nurses such as NPs and CNSs.

The CNS may use Rogers Diffusion of Innovation Model to implement an evidence-based protocol. Rogers Diffusion of Innovation Model consists of five key tenets in the systems sphere to include: relative advantage, compatibility, complexity, trialability, and observability. These tenets are scrutinized prior to the inception of an innovation to determine both its need and success. The three elements that are dependent on success of the diffusion theory are the innovation itself, communication, and time. The innovation must be built upon evidence and compiled from research for its credibility. Extensive collaboration amongst disciplines to determine the best type of communication for the innovation, in the most practical and cost effective manner is sought. Time refers to the rate of adoption of the innovation across the healthcare system.

The CNS is in a unique position to implement a PCSS scale for young adult athletes in the E.D. The advantage of implementing such a protocol and scale is evident in the growing concerns for misdiagnosed and mismanagement of those who have sustained a concussion as it is a growing public health concern. The PCSS scale can aid in identification of less severe symptoms and many times underreported symptoms such as a posttraumatic headache that can lead to increased morbidity during ones' lifetime if not identified. The CNS has the ability to experiment with the trialability of the PCSS scale with possibly clustering somatic symptoms to decrease the amount of time needed to complete the PCSS scale. A retrospective study can be completed to determine observability between time frames of induction of the PCSS protocol and scale with the previous years' concussive athletes. Collaborating amongst disciplines would be crucial to the success of this protocol and scale.

Nurse practitioners are more commonly being utilized in the acute care setting to include the E.D. With the help of the CNS a PCSS 
checklist can be implemented to increase awareness and timely assessment and diagnosis of postconcussive symptoms, namely PTH. However, with the increased awareness in the media and legislation appropriate education to both athletes and parents through selfefficacy can occur. Bandura's Self Efficacy Model encompasses personal, behavioral, and environmental factors into consideration. ${ }^{14}$

Personal factors may influence a postconcussive young adult athlete into seeking appropriate and timely cares include their belief system and perceived threat to their well being. Behavioral factors may include dedication to the team, status, importance of the athletes' performance, and risk taking behaviors. Environmental factors include the presence or absence of a certified athletic trainer, both coach and athletic trainers' knowledge of postconcussive symptoms, appropriate follow up, and management by the treating practitioner in the E.D. the athlete visits for symptom relief.

The NPs ability to distinguish initial postconcussive symptoms, namely PTH, which are commonly dismissed from protracted symptoms, is of utmost importance when assessing and managing a young adult athlete. ${ }^{15}$ Posttraumatic headaches may be synergistic and take on protracted courses of management. The athlete should be incorporated into the management plan and develop knowledge mastery of determining symptoms and appropriate management based on education received from the NP in the E.D. Bandura's Self Efficacy Model incorporates the patient at the center of care. The athlete's selfefficacy is the most important precondition for changing risk-taking behaviors. ${ }^{16}$

\section{Literature review}

A literature review was completed via PubMed and CINHL databases and GRADE criterion. Search terms singly and in combination indexed included: young adult, sports related concussion, mild traumatic brain injury, concussion, headache, posttraumatic headache, concussion symptoms, post concussive syndrome, postconcussion symptom scales, blunt head trauma, and trauma. The selection criteria for research-based articles included those with incidence and prevalence of concussion in athletes, symptoms of concussions occurring in sports, and PTH in athletes.

Benson et al. ${ }^{2}$ discovered PTHs was the most commonly reported debilitating symptom from sustaining a concussion in National Hockey League (NHL) athletes during a seven-year span from 1997 2004. The time loss associated with PTH ranged from zero to 346 days. ${ }^{2}$ However, each subsequent concussion injury per individual athlete increased their time loss of playing two fold. ${ }^{2}$ Posttraumatic headache was identified as a significant predictor of increased time loss greater than ten days. ${ }^{2}$ In addition, Benson and colleagues ${ }^{2}$ determined $11 \%$ of NHL athletes who had a previous documentation of concussion still had protracted symptoms, predominately a PTH.

Duhaime et al. ${ }^{15}$ analyzed helmeted collegiate athletes' clinical presentations of concussions from 3 universities over a four-year span. During this time athletes wore instrumented helmets with head impact telemetry (HIT) technology to record impact exposure. The HIT telemetry recorded frequency, location, and magnitude of all impact exposures. Duhaime et al. ${ }^{15}$ confirmed posttraumatic symptoms may occur from direct and indirect contact to both the head and bodies of athletes. Baseline testing of neurocognitive function was completed prior to the seasons on all athletes. Over half of all athletes diagnosed with a concussion had delayed onset of symptoms, mainly complaints of a PTH. Duhaime et al. ${ }^{15}$ examined diagnosis based off self reported symptoms and reliance of those self reported symptoms.
Baseline testing was performed at each individual academic institution by the certified athletic trainers. Testing included symptom checklists, computerized cognitive testing, known as immediate post-concussion assessment and cognitive test (imPACT), and at one institution a more in depth cognitive examination was completed via paper and pencil. Once a diagnosis of a concussion was documented regular and frequent intervals of neurocognitive testing occurred both at rest as well as during exertion. Based on the institution, sideline assessments of athletes varied in their completeness. However, symptom checklists were standardized across all three institutions both with immediate complaints of posttraumatic symptoms as well as delayed onset. These symptom checklists were utilized until all symptoms had resolved both at rest and during exertion.

Faux et al. ${ }^{17}$ examined the prevalence of PTH at one and three months post mild traumatic brain injury. Studies thus far have concentrated on the immediate effects following a concussion. Faux and colleagues ${ }^{17}$ queried on the type of PTH in the mild traumatic brain injury group over a short time interval. They sought to determine the differences of the headache types. Faux et al. ${ }^{17}$ suggest there may be a correlation between PTH and a psychological or stress reaction following a traumatic injury.

Faux et al. ${ }^{17}$ chose a minor injury group as their control group. It has been proposed young adult athletes may suffer a mild traumatic brain injury, mainly a concussion, from receiving a traumatic blow to any part of the body. Visual analog scales, the Galveston Orientation and Amnesia Test (GOAT), the River mead Post Concussion Symptoms Questionnaire, the modified Balance Error Scoring System (BESS), and the modified Rapid Screen of Concussion were used to collect data. Although multiple screening tools were completed, it is important to note the time to complete all tests was twenty minutes. The BESS test was not completed due to obvious safety issues by the control group as their injuries were orthopedic in nature. Telephone interviews took place at one and three month time intervals following initial presentation to the E.D. During the time of the interviews the River mead Post Concussion Symptoms Questionnaire was utilized. This questionnaire has been shown to be both valid and reliable in testing for posttraumatic symptoms. ${ }_{17}$

Upon initial presentation $100 \%$ of the mild traumatic brain injury group had PTH. Faux et al. ${ }^{17}$ determined that PTH occurred at both intervals following a traumatic injury, including those not sustaining a direct traumatic head injury. In fact, there was a slight increase in the percentage of those in the control group who reported a PTH at three months time. There were no demographic differences between both groups to include: age, level of education, and previous number of head injuries. It is suggested by Faux et al. ${ }^{17}$ PTH may be part of unresolved symptoms following a concussion affecting absenteeism from school, use of maladaptive chronic pain behaviors, and loss of quality of life.

Kontos et al. ${ }^{18}$ investigated the Post Concussive Symptom Scale (PCSS) on high school and collegiate athletes across the United States. The large sample size of 30,455 athletes completed the PCSS scale at baseline. One thousand four hundred thirty eight athletes completed the PCSS after sustaining a concussion. Kontos et al. ${ }^{18}$ sought to examine the PCSS factor structure and detect demographic differences in relation to sex and age that may affect scores. However, no correlation was found.

The PCSS is part of the imPACT computerized neuropsychological testing that assesses neurocognitive performance and identifies concussion symptoms. The PCSS uses a Likert-type scale outlining 
symptoms with their respective severity. The PCSS took approximately five minutes to complete per athlete. Post-concussion PCSS scales were completed individually in a quiet setting. Females reported more somatic symptoms at baseline and post-concussion compared to male athletes. Posttraumatic headache was the most common symptom reported post-concussion. The PCSS scale was determined to be very high in internal consistency and reliability for both healthy and concussed young adult student athletes.

Kontos et al. ${ }^{18}$ suggest that a PTH can be synergistic affecting other areas of the brain and symptoms to occur. This may explain the pathophysiologic connection between the metabolic crisis and the mismatch of energy and the demands placed on the brain after a concussion.

Lau et al. ${ }^{19}$ studied how predictive and at what point could the PCSS scale predict a protracted course after sustaining a concussion in high school athletes. One hundred seventy seven male athletes from 2002-2006 completed the imPACT computerized neuropsychological cognitive testing. A cutoff of $80 \%$ was shown to have a $73.1 \%$ positive predictive value (PPV) in predicting a protracted recovery course in this population. The cutoff scores showing statistically significant sensitivity was determined to be $80 \%$. In previous research imPACT testing has shown to be $81.9 \%$ sensitive and $89.4 \%$ specific. ${ }^{18}$ Almost half of all athletes tested were determined to have a protracted recovery course.

Although the PPV and sensitivity are relatively low for symptom clusters they may serve as an objective tool to guide practitioners in concussion management in the E.D. Lau et al. ${ }^{19}$ suggest predicting the length of recovery would greatly improve athletes with sports sustained concussion management. This may help nursing assess PTH in athletes through the use of the PCSS scale. Self reported symptoms of athletes who have had a concussion are highly relied upon for practitioners' management. This is why the importance of accurate detection and educating those recently involved in a traumatic injury has been publicized in the media. ${ }^{20}$

Lincoln et al..$^{20}$ examined 158,430 high school young adult student athletes to determine the incidence and relative risk of sustaining a concussion associated with twelve different female and male sports. Over the span of 11 years Lincoln et al. ${ }^{20}$ determined an alarming fourfold increase in incidence of concussions across all sports studied.

Certified athletic trainers across all schools were employed and mandated to document concussions and their symptoms into the school database for all injured athletes. Certified athletic trainers maintain certification through annual training by neuropsychologists specializing in concussion diagnosis and management. ${ }^{20}$ During 2005 there was a dramatic increase in the documentation of concussions and accompanying symptoms which may have in part been due to the increased employment of additional certified athletic trainers across all schools.

Lincoln et al. ${ }^{20}$ saw an increase in the number of concussions sustained by female student athletes across the 11-year span. The rate of concussions sustained in female sports similar to that of male sports was higher across the study period. Eleven percent of the 2651 concussions documented were repeat concussions from the athletes. ${ }^{20}$

Lincoln et al..$^{20}$ suggest the focus of concussions in athletes should not be limited to those traditionally associated with risk as both male and female athletes compete in similar sporting activities and female athletes tend to wear less protective gear. It is evident with increased incidence of concussions in young adult athletes concussions are a public health issue. ${ }^{11}$ Nursing may take the lead in the detection of post-concussion symptoms and educate athletes and parents alike. Advance practice nurses in the E.D. may help to educate families and patients on risk factors associated with repeated injury, such as with post concussive syndrome, second impact syndrome, red flags, when to return to the E.D., and the importance of follow up care. Nurse practitioners can refer concussed athletes to appropriate disciplines such as neuropsychologists, physical and occupational therapists, and pain specialists.

Lovell et al. ${ }^{21}$ examined 1,746 high school and collegiate athletes at baseline using the PCSS scale. This group of athletes completed the PCSS scale. Two hundred sixty concussed athletes completed the PCSS scale during three different time intervals to include: within 72 hours, 4-8 days, and 7-30 days. Lovell et al. ${ }^{21}$ found that PTH was the most commonly reported symptom across all athletes and time intervals. Although, symptoms decreased across time intervals, $5 \%$ of athletes experienced a worsening of PTH symptoms between seven and 30 days.

Register Mihalik et al. ${ }^{22}$ studied effects of preseason headaches and PTH in athletes following a concussion at more than 100 high schools and 14 colleges during different time intervals. There was a correlation between the number of previous concussions sustained and the duration of symptoms the athletes presented with. Posttraumatic headache was the dominant symptom complaint in almost $90 \%$ of athletes.

Register Mihalik et al. ${ }^{22}$ used the Graded Symptom Checklist and the Automated Neuropsychological Assessment Metrics and Standardized Assessment of Concussion exam to test neuropsychological cognitive function. The neuropsychological cognitive testing assessed for math processing, Sternberg memory, matching samples, continuous performance, and reaction times. These checklists and examinations were given at three different intervals to include: day one, day three, and day seven. Athletes who had baseline headaches were found to have sustained one or more concussions previously. These same athletes were shown to have protracted recovery periods and worsening symptoms across all time intervals

Lincoln et al. ${ }^{20}$ identified a clear indication for the need of increased awareness of concussions in young adult athletes. The incidence of concussions in athletes increased over an 11-year period. This increase is not probable to decline without the proper objective tools put into place and utilized in E.D.s. Although different postconcussion assessment scales and checklists were utilized in the previous studies the aim was to identify a valid and reliable objective tool identifying concussive symptoms. All studies except Lincoln et al. ${ }^{20} \&$ Register Mihalik et al. ${ }^{22}$ utilized the PCSS scale, which is a part of the imPACT neuropsychological cognitive battery of tests.

\section{Limitations}

Weakness of the previous studies included small sample sizes in isolated areas in the United States. Such small sample sizes decrease the generalizability of these studies results across the United States. Benson $^{2} \&$ Lovell et al. ${ }^{21}$ studies included males only. This too can decrease the generalizability of such studies. Additionally, utilized scales are limited in their nature to the honesty of athletes. Athletes may underreport or deny symptoms so they may return to playing their respective sports sooner. In several of the studies the acknowledgement of previous concussion injury was not collected which could have added an additional layer of knowledge to the symptoms of athletes and perhaps their protracted courses of recovery. 


\section{Implications for practice}

With the inception of California Assembly Bill 25 (AB25) the requirement of annual concussion education to both athletes and their parents is mandated prior to initiating practice or competition and requires the immediate removal of a concussed athlete from play and with evaluation by a licensed health care provider prior to returning to both school and their respective sports. ${ }^{23}$ With California AB25 implemented into law it is clear concussions in young adult student athletes are a public health concern. Posttraumatic headaches are the leading symptom ranging from $79-100 \%$ occurrence immediately status posts injury.

The incidence of concussed athletes seen in the E.D. has steadily increased over the last fifteen years. ${ }^{20}$ Implications for the acute care NP and CNS through implementation of an objective tool, such as the PCSS scale, may cut the risk of mild traumatic brain injury and concussion, decrease costs associated with protracted symptoms, decrease morbidity and mortality rates, decrease the time of lost school or work, decrease waste in the healthcare system by accurately assessing and diagnosing concussions based on common symptoms, and increase awareness and knowledge through the continuum of care for concussed athletes through a multidisciplinary and collaborative approach.

\section{Recommendations}

Currently there are no concrete evidence based guidelines determining when athletes are able to return to school or play their respective sports. The acute care NP and CNS in the E.D. may collaborate amongst disciplines to coordinate their efforts in implementing a PPCS scale. Among the multidisciplinary team are: the E.D. NP, CNS, and MDs, E.D. nursing staff, the trauma NP and CNS and MDs, physical, occupational, and speech therapists, case managers, social workers, neurologists, pain management, physiatrist, psychologists, and neuropsychologists. This multidisciplinary team is needed to adequately care for the young adult concussed athlete. ${ }^{8}$ Outside the healthcare system coaches, certified athletic trainers, school administrators, principals, school nurses, and counselors should be incorporated into the brainstorming and educational outreach within the community after initial implementation has occurred. The $\mathrm{CNS}$ is an excellent resource to consult within these groups outside of the healthcare system.

The sensitivity, specificity, and positive predictive value of PCSS scales have been documented in depth. As most concussed young adult athletes with complaints of PTH render their care and have initial contact with a practitioner in the E.D. ${ }^{24}$ It is appropriate to implement the PCSS scale in the E.D. where these patients are most commonly seen. ${ }^{24}$ Outcomes can be measured by the readmission rates of these patients in the E.D. Outcomes from follow up visits with detailed lists of specialists can occur to distinguish protracted courses of recovery from short recovery periods. This data can be collected retrospectively.

\section{Conclusion}

The PCSS scale has been proven valid, reliable, and to have a high PPV in accurately diagnosing concussive symptoms and may be able to predict protracted recovery courses in an acute care setting, such as in the E.D. ${ }^{5,15-19,21,22,24,25}$ These scales and checklists can be implemented among a multidisciplinary team by using Rogers Diffusion of Innovation theory. This may help identify obstacles early on for successful implementation of such checklists and scales. ${ }^{26}$ The importance of a clearly defined definition of concussion was identified across all studies analyzed. Although the definitions varied slightly in all studies, it was clear symptoms associated with concussed athletes were predominately a PTH. ${ }^{27}$

Concussions were shown not to be discriminatory in nature due to demographics such as sex and age and occurred in both male and female athletes amongst the various studies. ${ }^{28}$ Although PTH occurs immediately in approximately $79-100 \%$ of all traumatic injuries in young adult athletes it may have delayed onset and presentation. ${ }^{2}$ The sequelae accompanying PTH may lead to unresolved cognitive capacity and increased mortality rates amongst young adult athletes with repeated injury prior to the complete resolution of symptoms and pre-injury status. Both the NP and CNS have the abilities to collaborate amongst disciplines to advocate for this population and use evidenced based research and nursing theory to complement individualized management plans of those suffering from PTH. ${ }^{29}$

Future nursing research is needed to accurately detect the global impact PTH and concussions have on young adult athletes. Additional concussions incurred by athletes and their long term neuropsychological cognitive functioning may be greatly diminished. Future studies need to focus on the utilization of acute care NPs and CNSs in the E.D., their abilities to recognize concussive symptoms based on PCSS scales, education of nursing staff, and how their involvement may decrease system healthcare costs Tables $1-4$.

\begin{tabular}{|c|c|c|c|c|c|}
\hline Citation & Purpose & Sample/Setting & Methods & Results & Discussions \& limitations \\
\hline $\begin{array}{l}\text { Benson BW, } \\
\text { Meeuwisse, } \\
\text { WH, Rizos J, } \\
\text { Kang J, Burke, } \\
\text { CJ (20I I) A } \\
\text { prospective study } \\
\text { of concussions } \\
\text { among National } \\
\text { Hockey League } \\
\text { players during } \\
\text { regular season } \\
\text { games:The } \\
\text { NHL-NHLPA } \\
\text { Concussion } \\
\text { Program. } \\
\text { Canadian Medical } \\
\text { Association } \\
\text { Journal I83(8): } \\
905-9 \mid \text { I. }\end{array}$ & $\begin{array}{l}\text { Prospective } \\
\text { case series to } \\
\text { explore rates of } \\
\text { concussion, trends } \\
\text { r/t concussion in } \\
\text { the NHL, the initial } \\
\text { postconcussion s/ } \\
\text { sx's, PE findings } \\
\text { a\& time loss b/t } \\
\text { injury \& return to } \\
\text { play \& assessed } \\
\text { the utility of initial } \\
\text { postconcussive } \\
\text { manifestations in } \\
\text { predicting time } \\
\text { loss among NHL } \\
\text { hockey athletes } \\
\text { over } 7 \text { seasons } \\
\text { (I } 997-2004 \text { ) }\end{array}$ & $\begin{array}{l}\mathrm{n}=\mathrm{All} \text { NHL team } \\
\text { hockey athletes } \\
\text { during } 7 \text { seasons } \\
\text { from } 1997-2004 .\end{array}$ & $\begin{array}{l}\text { Univariable logistic } \\
\text { regression tested } \\
\text { for potential } \\
\text { predictors (s/ } \\
\text { sx's \& abn. Neuro } \\
\text { exam of time } \\
\text { loss. Multivariable } \\
\text { logistic regression } \\
\text { adjusted for age \& } \\
\text { position. Kruskal- } \\
\text { Wallis test for } \\
\text { median time loss } \\
\text { with recurrent } \\
\text { concussion } \\
\text { occurrence. }\end{array}$ & $\begin{array}{l}559 \text { concussions } \\
\text { during the } 7 \\
\text { year seasons. } \\
\text { Mean number } \\
\text { of concussions } \\
\text { annually was } 80 . \\
5.8 \text { concussions } \\
\text { per } 100 \text { players } \\
\text { per season. Time } \\
\text { loss ranged from } \\
0-342 \text { days. } 43 \% \\
\text { had longer than } \\
10 \text { days loss of } \\
\text { playing time. } \\
71 \% \text { reported } \\
\text { posttraumatic } \\
\text { headache (PTH). }\end{array}$ & $\begin{array}{l}\text { PTH was a significant predictor } \\
\text { of } \uparrow \text { length of time loss. Repeated } \\
\text { concussions } 2 \text { fold } \uparrow \text { time loss. } \\
\text { I I\% of PTH was still reported } \\
7 \text { years later. NHL athletes are } \\
\text { compensated \& therefore may } \\
\text { not have been completely honest } \\
\text { in their sx's. Underreporting of } \\
\text { concussions by the team MDs } \\
\text { may have occurred. Reporting } \\
\text { variance among MDs of various } \\
\text { teams could have occurred. } \\
\text { Onset of sx's not indicated. }\end{array}$ \\
\hline
\end{tabular}


Table Continued...

\begin{tabular}{|c|c|c|c|c|c|}
\hline Citation & Purpose & Sample/Setting & Methods & Results & Discussions \& limitations \\
\hline $\begin{array}{l}\text { Duhaime AC, } \\
\text { Beckwith JG, } \\
\text { Maerlender AC, } \\
\text { McAllister, TW, } \\
\text { et al. (20I2) } \\
\text { Spectrum of } \\
\text { acute clinical } \\
\text { characteristics } \\
\text { of diagnosed } \\
\text { concussions } \\
\text { in college } \\
\text { athletes wearing } \\
\text { instrumented } \\
\text { helmets. Journal } \\
\text { of Neurosurgery } \\
\text { I I } 7 \text { : I092-I099. }\end{array}$ & $\begin{array}{l}\text { Prospective study } \\
\text { analyzing the } \\
\text { spectrum of clinical } \\
\text { presentations } \\
\& \text { head impact } \\
\text { biomechanics used } \\
\text { by team medical } \\
\text { personnel to make } \\
\text { dx of sports- } \\
\text { related concussion } \\
\text { within a single } \\
\text { multisite study of } \\
\text { helmeted college } \\
\text { athletes. }\end{array}$ & $\begin{array}{l}\mathrm{N}=450 \text { athletes } \\
\text { participating in } \\
\text { contact sports } \\
\text { (football \& ice } \\
\text { hockey) from } \\
\text { Brown, Dartmouth, } \\
\text { \& Virginia Tech } \\
\text { Universities }\end{array}$ & $\begin{array}{l}\text { Clinical screening } \\
\text { battery, (symptom } \\
\text { reporting, } \\
\text { balance testing, } \\
\text { gene sampling, } \\
\text { \& neuroimaging) } \\
\text { \& Head impact } \\
\text { telemetry (HIT) } \\
\text { were analyzed } \\
\text { of athletes from } \\
2007-20 \text { I I }\end{array}$ & $\begin{array}{l}486,594 \text { head } \\
\text { impacts were } \\
\text { reported, } \\
48 \text { separate } \\
\text { concussions } \\
\text { were dx in } 44 \\
\text { athletes; } 29 \text { had } \\
\text { c/o headache } \\
\text { (HA); } 3 \text { I had an } \\
\text { identified impact; } \\
\text { I9/c immediate } \\
\text { sx's; I } 2 \text { /c } \\
\text { delayed sx's; } 38 \\
\text { had delay in dx } \\
\text { b/t } 10 \text { min.- } 4 \\
\text { days. }\end{array}$ & $\begin{array}{l}\text { Each of the } 3 \text { institutions had } \\
\text { certified athletic trainers at each } \\
\text { game \& had guidelines in place } \\
\text { for identification \& management } \\
\text { of concussions. Definition of } \\
\text { concussion was agreed upon at all } \\
3 \text { institutions. } \\
\text { Delayed onset of sx's\& delayed } \\
\text { dx of concussion in the majority } \\
\text { of athletes } \\
\text { Biomechanical correlates showed } \\
\text { significant variability. } \\
\text { BESS, gene sampling \& } \\
\text { neuroimaging not done at all sites. }\end{array}$ \\
\hline
\end{tabular}

\section{Citation}

\section{Discussions \& limitations}

\begin{tabular}{|c|c|c|c|}
\hline $\begin{array}{l}\text { Faux S, Sheedy } \\
\text { J (2008) A } \\
\text { prospective } \\
\text { controlled study } \\
\text { in the prevalence } \\
\text { of posttraumatic } \\
\text { headache following } \\
\text { mild traumatic } \\
\text { brain injury. Pain } \\
\text { Medicine } 9(8) \text { : } \\
\text { I00I-I0II. }\end{array}$ & $\begin{array}{l}\text { Prospective study } \\
\text { to establish the } \\
\text { prevalence of } \\
\text { posttraumatic } \\
\text { headache (PTH), } \\
\text { persisting } \\
\text { at } 3 \text { months } \\
\text { following mild } \\
\text { traumatic brain } \\
\text { injury (mTBI), } \\
\text { concussion. }\end{array}$ & $\begin{array}{l}\text { I00 sequential } \\
\text { admissions with } \\
\text { mTBI\& } 100 \text { control } \\
\text { group of minor non } \\
\text { deceleration injuries } \\
\text { at a Level } 2 \text { inner } \\
\text { city trauma center } \\
\text { in the E.D. in Sydney } \\
\text { Australia }\end{array}$ & $\begin{array}{l}\text { Chi-squared test } \\
\text { for categorical } \\
\text { variables, } \\
\text { t-tests for } \\
\text { nonparametric } \\
\text { equivalent, Mann- } \\
\text { Whitney U test } \\
\text { or continuous } \\
\text { variables, visual } \\
\text { analog scale (VAS) } \\
\text { rated pain levels } \\
\text { \& the Riverweed } \\
\text { Post Concussion } \\
\text { Symptoms } \\
\text { Questionnaire } \\
\text { comparing } \\
\text { symptoms prior } \\
\text { to mTBI. }\end{array}$ \\
\hline
\end{tabular}

Over $15 \%$ of those with $\mathrm{mTBI}$ had persistent PTH @ 3 months compared to $2 \%$ of minor non deceleration controls

Baseline testing revealed a 4 factor predominate symptoms. Posttraumatic headache was documented most frequently post-concussion. Females reported more symptoms at baseline \& post-concussion than males. No age differences were significant pre/post.
First Australian prospective study in prevalence of PTH following amTBI.

Control group asked about pain $\&$ the $\mathrm{mTBI}$ group asked about headache on VAS.

Control group had minor orthopedic injuries.

Initial presentation to the E.D. $100 \%$ of mobile patients had PTH.

Statistically significant proportion of mTBI group suffered with PTH @ 3 months \& the presence of post concussive syndrome.

Limitation was that the inclusion of previous concussion or mild traumatic brain injury was not noted. This study only looked at 7 day length of time after sustaining a concussion. Fluctuations in hormonal levels in females could have skewed the data with more females reporting baseline symptoms. Comparison in females could have been identified by asking if PTH was less, the same, or more severe after sustaining a concussion
PCSS scale. 


\begin{tabular}{|c|c|c|c|c|c|}
\hline Citation & Purpose & Sample/Setting & Methods & Results & Discussions \& limitations \\
\hline $\begin{array}{l}\text { Lau BC, Collins } \\
\text { MW, Lovell, } \\
\text { MR (20I2) } \\
\text { Cutoff scores in } \\
\text { neurocognitive } \\
\text { testing and } \\
\text { symptom clusters } \\
\text { that predict } \\
\text { protracted recovery } \\
\text { from concussions } \\
\text { in high school } \\
\text { athletes. Journal } \\
\text { of Neurosurgery } \\
70(2): 371-379 \text {. }\end{array}$ & $\begin{array}{l}\text { Prospective } \\
\text { cohort study } \\
\text { to examine } \\
\text { cutoff scores in } \\
\text { neurocognitive } \\
\text { \& PCSS scores } \\
\text { when classifying } \\
\text { protracted } \\
\text { recovery in } \\
\text { concussed high } \\
\text { school athletes } \\
\text { for predicting } \\
\text { prognosis. }\end{array}$ & $\begin{array}{l}\text { I } 77 \text { male high } \\
\text { school athletes } \\
\text { from a Pennsylvania } \\
\text { high school from } \\
\text { 2002-2006 }\end{array}$ & $\begin{array}{l}X^{2} \text { tests examined } \\
\text { differences b/t short } \\
\text { \& protracted groups } \\
\text { (preconcussionhx of } \\
\text { migraine or headache, } \\
\text { presence/absence } \\
\text { of ADHD, learning } \\
\text { disabilities, and } \\
\text { previous concussions. }\end{array}$ & $\begin{array}{l}58 \text { athletes had a } \\
\text { short recovery from } \\
\text { post-concussion } \\
\text { sx's. } 50 \text { athletes had } \\
\text { a protracted length } \\
\text { of recovery (> I4 } \\
\text { days). No statistical } \\
\text { significance b/t ages } \\
\text { PTH was most } \\
\text { reported in } 4 \text { out of } \\
8 \text { variables. Cutoff } \\
\text { determined at } 80 \% \\
\text { sensitivity. } 73.17 \% \text { PPV } \\
\text { for protracted sx's. }\end{array}$ & $\begin{array}{l}\text { Limitation includes a small } \\
\text { sample size. Generalizability } \\
\text { may not be possible as a } \\
\text { single institution in one state } \\
\text { with only male athletes was in } \\
\text { the cohort study. Concussions } \\
\text { \& their recovery length are } \\
\text { individualized. Prospects of } \\
\text { an objective tool to identify } \\
\text { those at risk for protracted } \\
\text { courses of recovery may aid } \\
\text { in management of athletes in } \\
\text { returning back to school \& } \\
\text { their perspective sports. }\end{array}$ \\
\hline
\end{tabular}

\begin{tabular}{lllll}
\hline Citation & Purpose & Sample/Setting & Methods
\end{tabular}

Lincoln AE, Caswell

SV, Almquist JL,

Dunn RE, Norris JB, et al. (20II) Trends in concussion

incidence in high school sports: A prospective II year study. American Journal of Sports Medicine 39(5): 958-963. GL, Collins MW, Podell K., Johnston KM, et al. (20l0) Measurement of symptoms following sportsrelated concussion: Reliability and normative data for the post-concussion Neuropsychology 13(3): I66-I74.
Lovell MR, Iverson scale.Applied

\section{Prospective} epidemiology study to examine the incidence \& relative risk of concussion in high school athletes. $\mathrm{n}=158,430$ student athletes in 12 males \& females' sports in 25 high schools from 1997-2008.

Prospective examine high school \& collegiate athletes and their prospective symptoms following a concussion across three time intervals with the concussion scale. cohort study to use of a post-
Retrospective study examining the effects of

Register Mihalik J, Guskiewicz KM, Mann JD, Shields EW (2007) The effects of headache of neurocognitive function. Clinical Journal of Sports Medicine 17(4): 282-288. on clinical measures preseason baseline headache \& PTH on neurocognitive function in high school \& collegiate athletes with a concussion over 3 different time intervals in a clinical athletic training setting \& sports medicine research laboratory
Chi-squared test for categorical variables, $t$-tests for nonparametric equivalents. Standardized Assessment of field assessment

$\mathrm{n}=\mathrm{I}, 746$ high school and collegiate athletes both male \& female across 15 high schools \& 10 colleges between Canada, Michigan \& Pennsylvania. N2=260 high school \& collegiate athletes sustaining a concussion.

$n=247$ concussed athletes in 110 high schools and I 4 colleges across the eastern United States over 3 time intervals (day I, day 3, \& day 7 ). Concussion for on-
265 I concussions were recorded. II\% was repeat concussion from the same athletes. Rate of concussions sustained increased by 4.2 fold, with an average annual increase of $15.5 \%$ across all sports studied. Girls showed $\mathrm{a} \uparrow$ rate of incidence of concussions compared to boys of similar sports.

There was a linear $\downarrow$ in total symptoms across the time intervals of within 72 hours, 4-8 days, \& 7-30 days. PTH was the most reported symptom \& one that remained across all 3 time intervals. There was a worsening of symptoms in $5 \%$ of the population in the 3rd time interval.

A Graded Symptom
Checklist was
used to assess sx's.
The Automated
Neuropsychological
Assessment Metrics
\& Standardized
Assessment of
Concussion was
used to examine
neuropsychological
function. BESS to
assess postural
balance.ANOVA
\&Turkey post-hoc
analysis for effects of
HA's \& to compare
specific group means.

Higher incidence of PTH at day I that periods. Those suffering from PTH reported an increased severity \& presence. Deficits in all neuropsychological cognitive testing was noted across all time intervals but improved by day 7 .
Certified athletic trainers were employed at all schools throughout the study period. In 2005 there were an increase in the number of certified athletic trainers across all schools in the study and may have skewed data collection. Limitation is that only one school district in one state was involved in the study but is one of the largest school districts in the country \& therefore may not be generalizable.

Most frequent symptom was posttraumatic headache at $88 \%$ of the concussed population. Internal consistency \& reliability of PCS is very high in both healthy \& concussed athletes. Limitation is that the time intervals overlapped \& were short in nature \& cld have skewed data. The postconcussion scale is a part of the imPACT computerized neurocognitive testing. Could the scale be used independently \& could that improve prognosis or protracted course.

Baseline headaches (HA) may little effect on neuropsychological testing.

Athletes with baseline HAs were noted to have 3 of more previous concussions. Athletes with baseline HAs may have a protracted recovery course $\&$ an increase in concussion symptoms. PTH is a sign of indicate other neurocognitive deficits. Limitations were that study population was declined over the study incomplete recovery \& may predominantly male. Limitation in the small sample size of study population. 


\section{Acknowledgements}

None.

\section{Conflict of interest}

The author declares no conflict of interest.

\section{References}

1. Centers for Disease Control and Prevention.

2. Benson BW, Meeuwisse WH, Rizos J, A prospective study of concussions among National Hockey League players during regular season games: The NHL-NHLPA Concussion Program. CMAJ. 2011;183(8):905-911.

3. McCrory P, Meeuwisse WH, Echemendia RJ, et al. What is the lowest threshold to make a diagnosis of concussion. British Journal of Sports Medicine. 2013;47(5):268-271.

4. U.S department of health and human services. Health, United States, 2008 with special feature on the health of young adults. Hyattsville, USA: Springer; 2008,

5. Collins MW, Field M, Lovell MR, et al. Relationship between postconcussion headache and neuropsychological test performance in high school athletes. Am J Sports Med. 2013;31(2):168-173.

6. Malisoux L, Frisch A, Urhausen A, et al. Monitoring of sport participation and injury risk in young athletes. Journal of Science and Medicine in Sport. 2013;16(6):504-508.

7. Reuben A, Sampson P, Harris AR, et al. Postconcussion syndrome (PCS) in the emergency department: Predicting and pre-empting persistent symptoms following a mild traumatic brain injury. Emergency Medicine Journal. 2013;31(1)

8. Stevens J. Traumatic brain injury. The Kansas Nurse. 2008;83(3):1-5

9. Menon DK, Schwab K, Wright DW, et al. Position statement: Definition of traumatic brain injury. Archives of Physical Medicine and Rehabilitation. 2010;91(11):1637-1640.

10. Babcock L, Byczkowski T, Wade SL, et al. Predicting post concussion syndrome after mild traumatic brain injury in children and adolescents who present to the emergency department. JAMA Pediatrics. 2013;167(2):156-161.

11. Butler IJ. Postconcussion syndrome after mild traumatic brain injury in children and adolescents requires further detailed study. JAMA Neurol. 2013;70(5):636-637.

12. Agency for Healthcare Research and Quality; 2009.

13. American College of Surgeons; 2012.

14. Bandura A. Self-efficacy: Toward a unifying theory of behavior change. Psychol Rev. 1977;84:191-215.
15. Duhaime AC, Beckwith JG, Maerlender AC, et al. Spectrum of acute clinical characteristics of diagnosed concussioins in college athletes wearing instrumented helmets. J Neurosurg. 2012;117(6):1092-1099.

16. Bandura A. Self-efficacy mechanism in human agency. American Psychologist. 1982;37:122-147.

17. Faux S, Sheedy J. A prospective controlled study in the prevalence of posttraumatic headache following mild traumatic brain injury. Pain Medicine. 2008;9(8):1001-1011.

18. Kontos AP, Elbin RJ, Schatz P, et al. A revised factor structure for the post-concussion symptom scale: Baseline and postconcussion factors. American Journal of Sports Medicine. 2012;40(10):2375-2384.

19. Lau BC, Collins MW, Lovell MR. Cutoff scores in neurocognitive testing and symptom clusters that predict protracted recovery from concussions in high school athletes. Neurosurgery. 2012;70(2):371-379.

20. Lincoln AE, Caswell SV, Almquist JL, et al. Trends in concussion incidence in high school sports: A prospective 11 year study. American Journal of Sports Medicine. 2011;39(5):958-963.

21. Lovell MR, Iverson GL, Collins MW, et al. Measurement of symptoms following sports-related concussion: Reliability and normative data for the post-concussion scale. Appl Neuropsychol. 2010;13(3):166-174.

22. Register Mihalik J, Guskiewicz KM, Mann JD, et al. The effects of headache on clinical measures of neurocognitive function. Clin J Sport Med. 2007;17(4):282-288.

23. Hayashi M, Buchanan, Conway. USA: California Assembly Bill; 2011.

24. Chen JK, Johnston KM, Collie A, et al. A validation of the post concussion symptom scale in the assessment of complex concussion using cognitive testing and functional MRI. Journal of Neural Neurosurgery and Psychiatry. 2007;78:1231-1238.

25. Piland SG, Motl RW, Ferrara MS, et al. Evidence for the factorial and construct validity of a self-report concussion symptoms scale. J Athl Train. 2003;38(2):104-112.

26. McKinley MG. Evolution of the clinical nurse specialist in acute and critical care. In: MG McKinley editor. Acute and Critical Care Clinical Nurse Specialist. Netherlands: Saunders Elsevier; 2007. p. 1-11.

27. Piebes SK, Snyder AR, Bay RC, et al. Measurement properties of headache-specific outcomes scales in adolescent athletes. J Sport Rehabil. 2011;20(1):129-142.

28. Talavage TM, Nauman E, Breedlove EL, et al. Functionally-detected cognitive impairment in high school football players without clinicallydiagnosed concussion. Journal of Neurotrauma. 2010;31(4):327-338.

29. Vargas BB, Dodick DW. Posttraumatic headache. Current Opinion in Neurology. 2012;25(3):284-289. 